\title{
CARCINOMA OF BREAST- A STUDY PROFILE
}

\author{
Yashwant R. Lamture1, Balaji Salunke2, Shahabuddin $\mathrm{Md}^{3}$
}

1 Professor, Department of General Surgery, JNMC, Wardha, Maharashtra, India.

2 Assistant Professor, Department of General Surgery, JNMC, Wardha, Maharashtra, India.

3Junior Resident, Department of General Surgery, JNMC, Wardha, Maharashtra, India.

\begin{abstract}
BACKGROUND
Breast cancer is the most common site-specific cancer in women and is the leading cause of death from cancer for women of age 40 to 44 years. It is the second most common cancer in Indian women next only to carcinoma cervix. It is one of the commonest causes of death in many developed countries and becoming frequent in developing countries as well.

Aims and Objectives of Study-

1. To study the clinical presentations and epidemiological factors of patients with carcinoma breast in rural setup.

2. To study the efficacy of Surgery, followed by chemotherapy and hormonal therapy in carcinoma breast.

3. To study the pattern of recurrences and relation of recurrence with compliance to treatment.
\end{abstract}

ABSTRACT

\section{MATERIALS AND METHODS}

The present study was a prospective study carried out at Sushrut Hospital, Ahmedpur. Study duration was 3 years. Around 83 patients with untreated breast carcinoma were included.

\section{RESULTS}

All the 83 patients had lump in breast as their complaint and 13 patients had pain as their chief complaint. No patient in our study had any nipple discharge. All the patients with operable breast cancer were treated with modified radical mastectomy followed by chemotherapy and Tab. Tamoxifen $10 \mathrm{mg}$ twice daily. During follow-up, 2 patients had local recurrence. 4 were having distant metastasis with deaths during 3 years study duration.

\section{CONCLUSION}

Patients with carcinoma breast, presenting to hospital in rural setup tend to present late when their disease is either locally advanced or has spread widely. Most of the patients presenting at our setup belonged to stage III.

\section{KEY WORDS}

Cancer, Histopathology, Chemotherapy.

HOW TO CITE THIS ARTICLE: Lamture YR, Salunke B, Shahabuddin M. Carcinoma of breast- a study profile. J. Evolution Med. Dent. Sci. 2018;7(45):4857-4861, DOI: 10.14260/jemds/2018/1082

\section{BACKGROUND}

Breast cancer is the most common site-specific cancer in women and is the leading cause of death from cancer for women aged 40 to 44 years. It is the second most common cancer in Indian women next only to carcinoma cervix. It is one of the commonest causes of death in many developed countries in middle-aged women and is becoming frequent in developing countries as well. ${ }^{1}$ A total of 1,399,790 new cancer cases and 564,830 deaths from cancer are expected in the United States in 2006. When deaths are aggregated by age, cancer has surpassed heart disease as the leading cause of death for those younger than age 85 since 1999. Delayadjusted cancer incidence rates stabilised in men from 1995 through 2002, but continued to increase by $0.3 \%$ per year from 1987 through 2002 in women. Between 2002 and 2003, the actual number of recorded cancer deaths decreased by

'Financial or Other Competing Interest': None.

Submission 19-09-2018, Peer Review 18-10-2018,

Acceptance 23-10-2018, Published 05-11-2018.

Corresponding Author:

Dr. Balaji Salunke,

Assistant Professor,

Department of General Surgery,

JNMC, Wardha,

Maharashtra, India.

E-mail: balajisalunke04@gmail.com

DOI: $10.14260 /$ jemds/2018/1082
778 in men, but increased by 409 in women, resulting in a net decrease of 369 , the first decrease in the total number of cancer deaths since national mortality record keeping was instituted in 1930. The death rate from all cancers combined has decreased by $1.5 \%$ per year since 1993 among men and by $0.8 \%$ per year since 1992 among women. The mortality rate has also continued to decrease for the three most common cancer sites in men (lung and bronchus, colon and rectum, and prostate) and for breast and colon and rectum cancers in women. Lung cancer mortality among women continues to increase slightly. In analyses by race and ethnicity, African-American men and women have $40 \%$ and $18 \%$ higher death rates from all cancers combined than White men and women, respectively. Cancer incidence and death rates are lower in other racial and ethnic groups than in Whites and African-Americans for all sites combined and for the four major cancer sites. However, these groups generally have higher rates for stomach, liver and cervical cancers than Whites. Furthermore, minority populations are more likely to be diagnosed with advanced stage disease than are Whites. Progress in reducing the burden of suffering and death from cancer can be accelerated by applying existing cancer control knowledge across all segments of the population. The mortality rates from cancer breast have increased during the past 60 years in every country. In the 1970s, the probability of a woman in the United States 
developing breast cancer was estimated at 1 in 13 , in 1980 it was 1 in 11 and in 2002 it was 1 in 8.1.2

Halsted and Meyer advised dissection of axillary lymph node level 1 and 3. Both routinely resected the long thoracic nerve and the thoracodorsal neurovascular bundle with the axillary contents. ${ }^{3}$ Robert McWhirter described the technique of postoperative external beam radiation. ${ }^{4}$ Stafford warren, a radiologist at the University of Rochester, pioneered clinical mammography. ${ }^{5}$ Sir Geoffrey Keynes published the series of patient treated by wide local excision supplement with radium needles. ${ }^{6}$ Manchester staging system of carcinoma breast came into practice in 1940 and it was developed by Paterson. ${ }^{7}$ Madden, Auchincloss and Baker developed the operative technique, in which pectoralis minor muscle was left intact with modified radical mastectomy. ${ }^{8}$ However, medical therapies (adjuvant Chemotherapy, neoadjuvant chemotherapy and hormonal therapy) are now an important component of the treatment of almost all stages of invasive breast cancer. The introduction of film screen mammography took place in 1972 and in the same year John Wolfe made xeromammography commercially available. ${ }^{9}$ Cooper advised combination chemotherapy for advanced carcinoma of breast. ${ }^{10}$ Snyderman was the first to use silastic gel prosthesis to reconstruct the breast mound. ${ }^{11}$ Smalley et al and Blumenschein et al 1975 started FAC regime (5-Fluorouracil, Adriamycin, Cyclophosphamide) with improved survival rates. ${ }^{12}$

With all these various aspects in mind, we decided to study a series of 80 patients of breast cancer attending Sushrut Hospital, Ahmedpur. Analysis of our experience with respect to various aspects of breast cancer such as epidemiological influences, natural history, clinical features, management and survival of the patients has been presented.

\section{Aims and Objectives of Study}

1. To study the clinical presentations and epidemiological factors of patients with carcinoma breast in rural setup.

2. To study the efficacy of Surgery, followed by chemotherapy and hormonal therapy in Carcinoma breast.

3. To study the pattern of recurrences and relation of recurrence with compliance to treatment.

\section{MATERIALS AND METHODS}

Study Design- Present study was a cohort study.

Study Setup- Sushrut Hospital, Ahmedpur.

Study Duration- 3 years.

Around 83 patients with untreated breast carcinoma were entered. All the information about the patients was collected. Detailed histories and clinical examinations of all these patients were recorded. Following this, a provisional diagnosis was kept, and patients were subjected for investigations. Routine investigations done were complete haemogram, blood sugar, kidney function tests, liver function tests, blood grouping and $\mathrm{Rh}$ typing, urine examination, $\mathrm{x}$-ray chest and ultrasonography of abdomen and pelvis. Similarly, ECG and radiograms of other bones were done as and when indicated. The diagnosis was confirmed with biopsy.
Patients were then staged according to the standard TNM classification. In our study, we divided all the patients between operable and inoperable groups. Patients with stage I, II and IIIA were included in the operable group and stage IIIB, $C$ and IV were included in inoperable group. ${ }^{13}$

All the patients with operable breast cancer were treated with Modified radical mastectomy followed by chemotherapy and hormonal therapy. All the patients with inoperable breast cancer were treated with simple mastectomy followed by chemotherapy and then hormonal therapy.

After completion of six cycles of chemotherapy, patients were asked to come for follow-up once every three months for first year, six months for next year and yearly during subsequent years.

At each follow-up visit, examination was done to look for loco regional recurrence and distant metastasis. Patients presenting local recurrence or distant metastasis during the follow-up were given radiotherapy or chemotherapy respectively, depending on the site of recurrence.

\section{RESULTS}

All the 83 patients had lump in breast as their chief complaints and 13 patients had pain as their chief complaints. No patient in our study had any nipple discharge.

In our study, we divided all the patients between operable and inoperable groups. All the patients with operable breast cancer were treated with Modified radical mastectomy followed by chemotherapy and Tab. Tamoxifen $10 \mathrm{mg}$ twice daily.

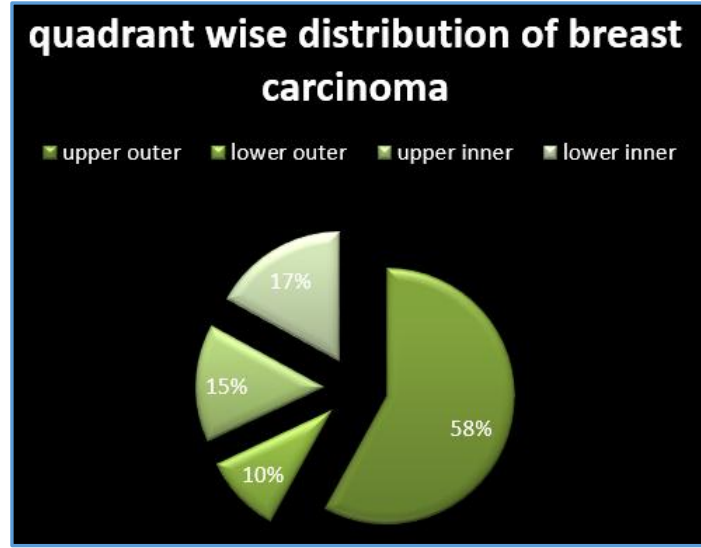

Figure 1. Upper Outer Quadrant was most Common Site of Carcinoma Breast

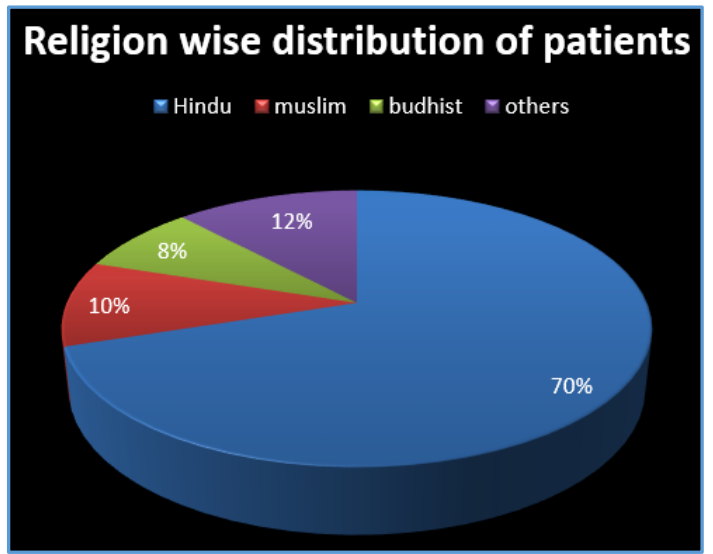

Figure 2. Religion wise Hindus were most commonly affected followed by Muslims 


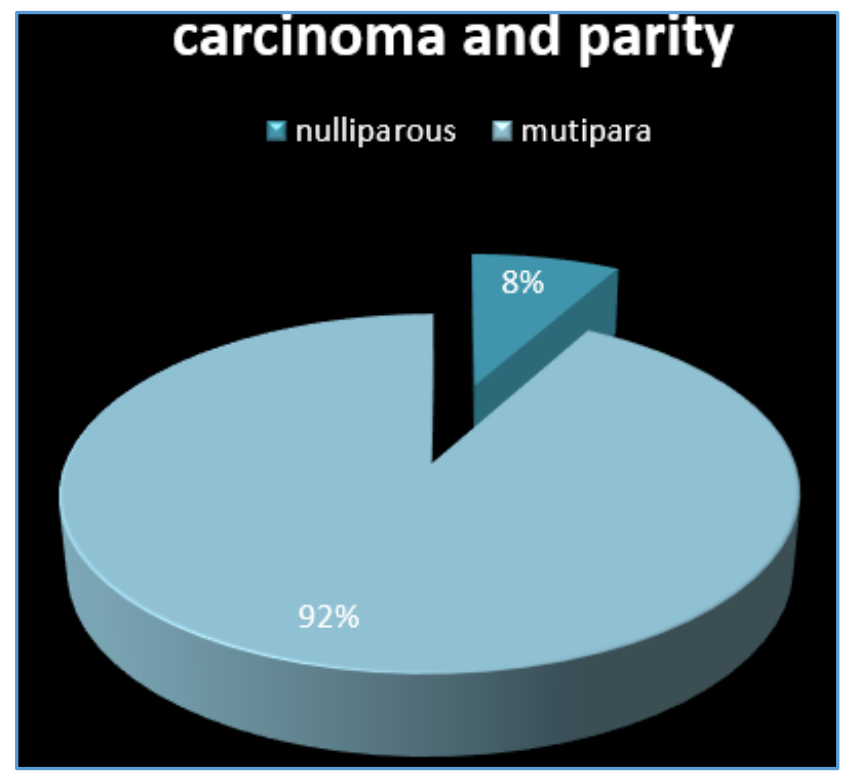

Figure 3. Incidence of Breast Carcinoma in relation to Parity

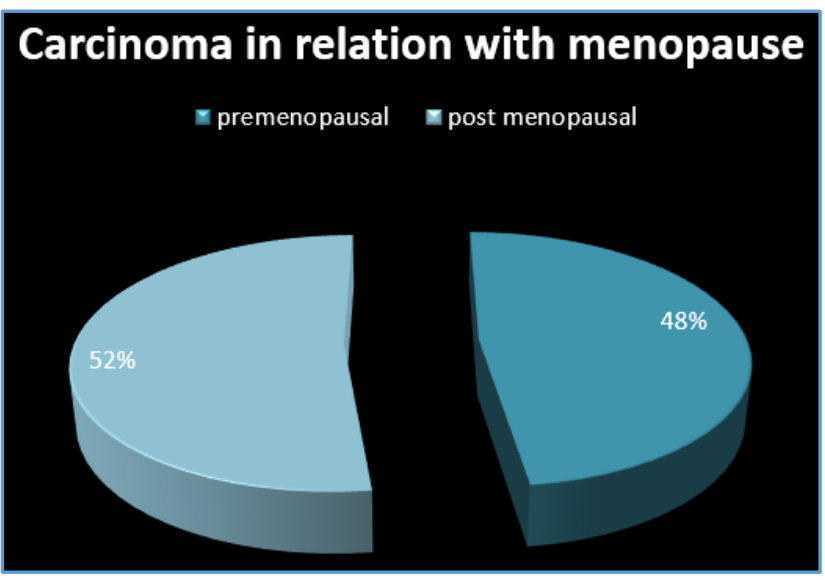

Figure 4. Relation to Menopause

$48 \%$ of female cases were having premenopausal status and $52 \%$ of female cases were post-menopausal. One case belongs to male gender.

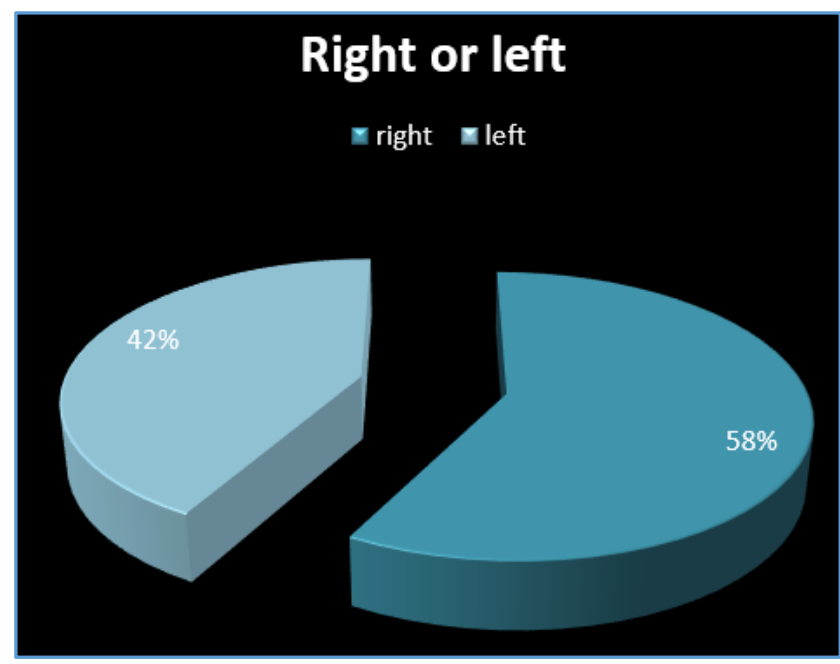

Figure 5. Incidence of Breast Carcinoma in Right and Left Breast

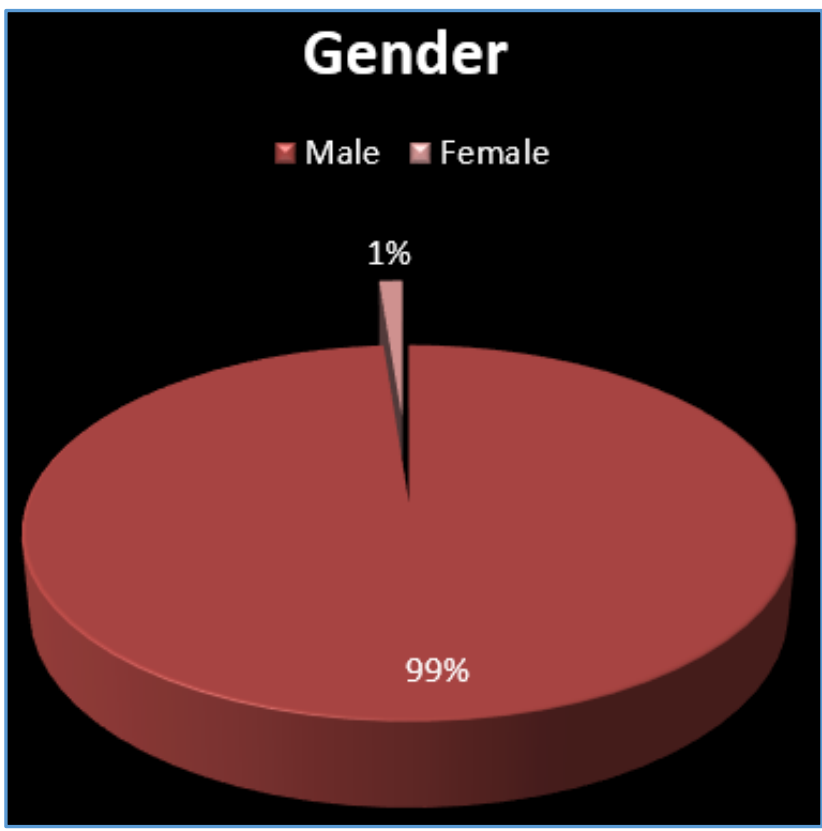

Figure 6. Incidence of Breast Carcinoma according to Sex

Incidence was thus more in the right than in the left breast in our study.

Histopathological examination showed that 82 out of 83 cases had invasive ductal carcinoma and only 1 patient had invasive lobular carcinoma in our study. No case with carcinoma in situ was seen.

Receptor studies were done in 17 post-menopausal cases, out of which 10 cases were positive for both oestrogen and progesterone.

\begin{tabular}{|c|c|c|}
\hline Site of Recurrence & No. of Cases & No. of Deaths \\
\hline Local & 2 & 0 \\
\hline Liver & 1 & 0 \\
\hline Lungs, Pleura & 2 & 2 \\
\hline Spine & 1 & 0 \\
\hline \multicolumn{3}{|c|}{ Table 1 } \\
\hline
\end{tabular}

In our study, lung metastasis was the chief cause of mortality. All those cases that had local recurrence or distant metastasis belonged to either inoperable group or were irregular in follow-up for chemotherapy.

\section{DISCUSSION}

As the range of options for the treatment of patients with breast cancer widens, so it becomes increasingly important that the clinician is provided with accurate prognostic information on which base therapeutic decisions can be taken.

All the 83 patients had lump in breast as their chief complaints and 13 patients had pain as their chief complaints. It comprises only one male patient and rest were from female gender. In our study, we divided all the patients between operable and inoperable groups. All the patients with operable breast cancer were treated with Modified radical mastectomy followed by chemotherapy and Tab. Tamoxifen $10 \mathrm{mg}$ twice daily. All patients were advised to take Tamoxifen in combination with chemotherapy on the basis of following study. 
On June 1, 1992, the National Surgical Adjuvant Breast and Bowel Project (NSABP) implemented a randomised clinical trial to evaluate the worth of tamoxifen for the prevention of breast cancer in women who were considered to be at increased risk for the disease. Although, tamoxifen prevented the appearance of a substantial number of breast cancers over the duration of study, the term "prevention" does not necessarily imply that the initiation of breast cancers has been prevented or that the tumours have been permanently eliminated. The primary aim of the NSABP Breast Cancer Prevention Trial (BCPT; P-1) was to determine whether tamoxifen administered for at least 5 years prevented invasive breast cancer in women at increased risk. Secondary aims were to determine whether tamoxifen administration would lower the incidence of fatal and nonfatal myocardial infarctions and reduce the incidence of bone fractures. Additional objectives were to evaluate breast cancer mortality and tamoxifen's adverse effects in order to assess the benefits and risks from the drug and in keeping with recent advances to obtain information with regard to breast cancer. Tamoxifen was chosen as the agent to be evaluated because of its demonstrated benefit when used alone as well as in combination with chemotherapy to treat advanced breast cancer and because of its proven efficacy in reducing tumour recurrence and prolonging survival when administered as postoperative adjuvant therapy in stages I and II disease. Findings indicating that tamoxifen-treated patients had a statistically significantly lower incidence of contralateral breast cancer and that most patients used tamoxifen safely with good compliance and minimal side effects also provided justification for its evaluation as a preventive agent. Equally compelling was the extensive information related to the drug's pharmacokinetics, metabolism and antitumor effects that had been observed in experimental animals and humans. In addition, there was evidence to indicate that tamoxifen interfered with the initiation and promotion of tumours in experimental systems and inhibited the growth of malignant cells by a variety of mechanisms. ${ }^{14}$

Hindus were most commonly affected religion followed by Muslims and Buddhists. Reason of this distribution was as demographically Hindu's were most populous in this area. Religion wise distribution in study done by Chopra et al was similar to our study. Giordano et al has mentioned that males account for less than $1 \%$ of all cases of breast cancer. This is comparable with our study.

Histopathological examination showed that 82 out of 83 cases had invasive ductal carcinoma and only 1 patient had invasive lobular carcinoma in our study. No case with carcinoma in situ was seen.

Chemotherapy was given to all the patients with breast cancer, except those patients of Stage I, who had no positive lymph nodes on histopathology report. There were only one such patients. So chemotherapy was given to 82 out of 83 cases. Out of 82 patients that were offered chemotherapy, 55 patients completed 6 cycles of chemotherapy, accounting for $66 \%$ of cases. However, remaining 27 were lost during follow-up.

So the total number of cases who are regular for followup and have either completed six cycles or are under chemotherapy are 55, accounting for $66 \%$ of good compliance. The remaining 27 patients out of 82 cases who were offered chemotherapy were either lost during follow-up without completing 6 cycles of chemotherapy or were irregular in follow-up and hence did not complete 6 cycles as advised. One case which was not offered any chemotherapy, was under regular follow-up.

After completion of six cycles of chemotherapy, patients were asked to come for follow-up once every three months for first year, six months for next year and yearly during subsequent years.

At each follow-up visit, examination was done to look for loco regional recurrence and distant metastasis. Total duration of follow-up in our study ranged from six months to 3 years.

During follow-up, 2 patients had local recurrence. All these patients had inoperable breast cancer at the time of presentation. Both these 2 patients belonged to stage IIIb. One had taken only 3 cycles of chemotherapy and 1 case received only 4 cycles. They were irregular for follow-up and presented only when they had local recurrence. We referred both of them for radiotherapy. Single case had associated secondaries in liver and another one secondaries in spine (See Table 1). All the cases with operable breast cancer, who were treated with modified radical mastectomy followed by chemotherapy and later hormonal therapy, and were under regular follow-up thereafter, are disease free at present and doing well. One case with Stage IIIb cancer, after completion of 6 cycles of chemotherapy and under regular follow-up presented with secondary in lumbar spine. She was referred for radiotherapy. Second case with stage IIIb at the time of presentation had only taken 1 cycle of chemotherapy and was not compliant thereafter. She presented one year later with multiple liver metastasis. She was then given 2 cycles of chemotherapy along with hormonal therapy. However, she was lost for follow-up thereafter. Another cases developed lung metastasis in follow-up after completing 6 cycles. They were under regular follow-up. One had stage IIIb cancer and another stage IV cancer at the time of initial presentation. Both these patients died in the Surgery wards. These were the only two deaths recorded in our study. There was only one case with operable breast cancer in our study, who developed metastasis in follow-up, two years after surgery. She had stage IIIa cancer at the time of presentation. However, she took only one cycle of chemotherapy and after that presented only two years later with brain metastasis.

\section{CONCLUSION}

Patients with carcinoma breast, presenting to hospital in rural set-up tend to present late, when their disease is either locally advanced or has distant metastasis. Most of the patients presenting at our set-up belonged to stage III. In our study, most of the patients belonged to age group of $40-49$ years. Carcinoma breast was most often seen on right side and in upper outer quadrant. Histopathologically, most had invasive ductal carcinoma. Modified radical mastectomy is the surgical procedure of choice in operable breast cancer. Carcinoma breast patients, if presenting early and are compliant to treatment and regular for follow-up have a long disease-free life. Those patients who were regular for followup, which ranged from 6 months to 4 and a half years, and who were compliant with chemotherapy and hormonal therapy, and belonged to operable group had no local recurrence or distant metastasis in our study. In our study, 
recurrence was seen only in those patients who had inoperable malignancy at the onset or who were noncompliant for chemotherapy and hormonal therapy and were irregular for follow-up. Lung metastasis was the chief cause of mortality in this study.

In order to have good results, patients should report early. This can be done by educating people about self-breast examination and making them aware of the disease.

\section{ACKNOWLEDGEMENTS}

Authors would like to acknowledge to Surgery Department of Sushrut Hospital for kind co-operation.

\section{REFERENCES}

[1] Guinee VF. Epidemiology of breast cancer. In: Bland KI, Copeland EM 3rd, eds. The breast: comprehensive management of benign malignant diseases. Philadelphia: WB Saunders 1998: p. 339.

[2] Jemal A, Murray T, Samuels A, et al. Cancer statistics, 2003. CA Cancer J Clin 2003;53(1):5-26.

[3] Halsted WS. The results of operations for the cure of cancer of the breast performed at the Johns Hopkins Hospital from June 1889 to January 1894. Johns Hopkins Hosp Rep 1894;4:297-350.

[4] Rodger A. The great 1948 debate: Sir Gordon-Taylor versus Robert McWhirter - Mcwhirter's challenge to the surgical gold standard. ANZ Journal of Surgery 2002;72 Supple 1:A9.

[5] Andolina V, Lille S, Willison KM. Mammographic imaging: a practical guide. Lippincott Williams \& Wilkins 2001: p. 4.

[6] Bomford CK, Kunkler IH. Walter and Miller's textbook of radiotherapy: radiation physics, therapy and oncology. $6^{\text {th }}$ revised edn. London, UK: Elsevier Health Sciences 2002: p. 438-41.
[7] Wapnir IL, Wartenberg DE, Greco RS, et al. Three dimensional staging of breast cancer. Breast Cancer Research and Treatment 1996;41(1):15-9.

[8] Auchincloss H. Significance of location and number of axillary metastasis in carcinoma of the breast: a justification for a conservative operation. Ann Surg 1963;158:37-46.

[9] Carlson RW, Favret AM. Multidisciplinary management of locally advanced breast cancer. The Breast Journal 1999;5(5):303-7.

[10] Huggins C, Bergenstal DM. Inhibition of human mammary and prostatic cancer by adrenalectomy. Cancer Res 1952;12(2):134-41.

[11] Sickles EA. Wolfe mammographic parenchymal patterns and breast cancer risk. American Journal of Roentgenology 2007;188:301-3.

[12] Smalley RV, Carpenter J, Bartolucci A, et al. A comparison of cyclophosphamide, adriamycin, 5fluorouracil (CAF) and cyclophosphamide, methotrexate, 5-fluorouracil, vincristine, prednisone (CMFVP) in patients with metastatic breast cancer: a Southeastern Cancer Study Group project. Cancer 1977;40(2):625-32.

[13] Krag DN, Weaver DL, Alex JC, et al. Surgical resection and radiolocalization of the sentinel lymph node in breast cancer using a gamma probe. Surg Oncol 1993;2(6):335-40.

[14] Chopra B, Kaur V, Singh K, et al. Age shift: breast cancer is occurring in younger age groups - Is it true? Clin Cancer Investig Journal 2014;3(6):526-9. http://www.ccijonline.org/text.asp?2014/3/6/526/142652 